Quim. Nova, Vol. 36, No. 9, 1464-1467, 2013

\title{
MINIPROJETO PARA ENSINO DE QUÍMICA ORGÂNICA EXPERIMENTAL BASEADO NO ACOPLAMENTO CATALÍTICO N-C PROMOVIDO POR MICRO-ONDAS
}

\author{
Michelle Dalmás, Neusa Fernandes de Moura e Gilber Ricardo Rosa* \\ Escola de Química e Alimentos, Universidade Federal do Rio Grande - FURG, Campus Santo Antônio da Patrulha. Rua Barão do \\ Cahy, 125, Cidade Alta, 95500-000 Santo Antônio da Patrulha - RS, Brasil \\ Cristina Lorenski Ferreira, João Augusto Oliveira dos Santos e Thais Kazakevicius Bolzan \\ Curso Técnico em Química, Colégio Dom Feliciano. Av. Dr. José Loureiro da Silva, 655, Centro, 94010-001 Gravataí - RS, Brasil \\ Fernando Kokubun \\ Instituto de Matemática, Estatística e Física, Universidade Federal do Rio Grande - FURG, Campus Santo Antônio da Patrulha. \\ Rua Barão do Cahy, 125, Cidade Alta, 95500-000 Santo Antônio da Patrulha - RS, Brasil
}

Recebido em 7/6/13; aceito em 1/7/13; publicado na web em 9/8/13

\begin{abstract}
MINI-PROJECT FOR TEACHING EXPERIMENTAL ORGANIC CHEMISTRY BASED ON N-C CROSS-COUPLING PROMOTED BY MICROWAVE. This paper describes a three-week mini-project for an Experimental Organic Chemistry course. The activities include $\mathrm{N}-\mathrm{C}$ cross-coupling synthesis of $\mathrm{N}$-(4-methoxyphenyl) benzamide in an adapted microwave oven by a copper catalyst (CuI). Abilities and concepts normally present in practical organic chemistry courses are covered: use of balances, volumetric glassware, separation of mixtures (liquid-liquid extraction and filtration), chromatographic techniques, melting point determination and stoichiometric calculations.
\end{abstract}

Keywords: N-C cross-coupling; microwave; chemical education.

\section{INTRODUÇÃO}

A síntese de compostos orgânicos contendo a ligação N-C tem sido muito investigada pela indústria de química fina em função de suas interessantes aplicações biológicas. Tais compostos destacam-se como inibidores enzimáticos, ${ }^{1,2}$ agentes cardiotônicos, ${ }^{3}$ agentes anti-glaucoma, ${ }^{4}$ além de apresentarem atividade fungicida e herbicida. ${ }^{5,6}$ Dentre a vasta gama de compostos nitrogenados bioativos encontra-se a família das benzamidas N-substituídas com importantes aplicações no campo da agroquímica ${ }^{5,6}$ e medicina. ${ }^{7}$

Focando-se somente no segmento da agroquímica, em 2011, as vendas de agrotóxicos no país alcançaram cerca de $\mathrm{R} \$ 14$ bilhões, um aumento de mais de $72 \%$ entre 2006 e 2012, e o consumo médio por hectare saiu de 7 para 10,1 quilogramas, num salto de 43,2\%. Desde 2008, o Brasil ocupa a primeira posição no consumo mundial, quando ultrapassou os Estados Unidos. ${ }^{8}$

Assim, a importância do tema (síntese de moléculas contendo ligação $\mathrm{N}-\mathrm{C}$ ) enquanto conteúdo em disciplinas experimentais de química orgânica é inquestionável. No entanto, são raros os experimentos que englobem a síntese de derivados da benzamida em cursos de graduação e, mais escassos ainda, os que trabalham com esse tema relacionado a acoplamento catalítico.

$\mathrm{Na}$ última década houve um aumento significativo no uso de aquecimento por micro-ondas em artigos de síntese orgânica publicados por brasileiros respaldado, principalmente, nos baixos tempos reacionais empregados. ${ }^{9}$ Esse dado segue uma tendência mundial visto que a técnica proporciona protocolos reacionais mais "verdes" que, inicialmente, eram buscados via substituição de reagentes e/ou solventes ou com reaproveitamento de biomassa, visando condições reacionais mais amigáveis ao meio ambiente..$^{10,11}$ Fixando-se no trabalho de grupos de pesquisa brasileiros usuários de micro-ondas, vemos as contribuições na educação em química de Santos e colaboradores no emprego de micro-ondas doméstico (sem adaptações) em aula

*e-mail: gilberrosa@furg.br prática de nitração do saliciladeído; ${ }^{12}$ e de Konrath e colaboradores em aula prática de síntese de fenitoína em micro-ondas dedicado. ${ }^{13}$

Partindo do pressuposto que em cursos de graduação deve-se trabalhar com temas atuais e de relevância na formação do aluno, propomos o referido trabalho que trata da síntese de derivados da benzamida via acoplamento catalítico catalisado por cobre e aquecido por micro-ondas. Tal tema, em nossa concepção, é imprescindível na formação de qualquer estudante de química, sendo aqui apresentado na forma de miniprojeto com duração de 3 aulas. ${ }^{14} \mathrm{O}$ termo miniprojeto será usado para descrição das atividades envolvendo todo o processo de obtenção do derivado da benzamida, sendo que tal artifício didático já fora trabalhado por nosso grupo numa outra disciplina gerando resultados animadores. ${ }^{15}$

Na primeira aula, os alunos fazem a reação da benzamida com o 4-iodoanisol catalisada por $\mathrm{CuI}$ em forno de micro-ondas doméstico adaptado (Esquema 1). ${ }^{16}$

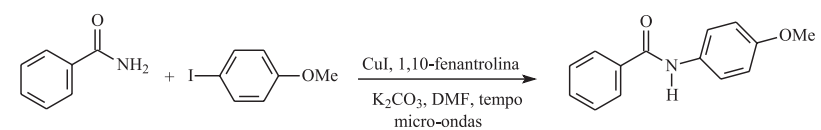

Esquema 1. Reação de acoplamento catalítico N-C estudada

A separação do produto ocorre na segunda aula através de extração com solventes e cromatografia em camada delgada e/ou cromatografia em coluna. Já na terceira aula, é purificado o produto no rotaevaporador e identificado por ponto de fusão.

\section{PARTE EXPERIMENTAL}

\section{Materiais}

Para a execução das 3 aulas, para cada grupo de alunos, serão necessários balões de fundo redondo de 25 e $250 \mathrm{~mL}$ com junta 24/40 (um de cada), esferas de ebulição, copos de béquer de $50 \mathrm{~mL}$ e 100 $\mathrm{mL}$ (um de cada), um funil de decantação de $100 \mathrm{~mL}$, um termômetro 
com escala de -10 até $+310^{\circ} \mathrm{C}$, espátulas, vidros de relógio, provetas de 15 e $100 \mathrm{~mL}$ (uma de cada), uma pipeta graduada de $5 \mathrm{~mL}$, uma coluna cromatográfica de $30 \mathrm{~cm}$ de comprimento e diâmetro interno de $2 \mathrm{~cm}$, estante com 25 tubos de ensaio, cromatoplacas de sílica gel $60 \mathrm{~F}_{254}$, uma cuba cromatográfica.

\section{Reagentes}

Os reagentes e solventes a serem usados são benzamida, 4-iodoanisol, carbonato de potássio anidro, iodeto cuproso, 1,10-fenantrolina, DMF (N,N-dimetilformamida), sílica gel 60 (70-230 mesh), hexano, acetato de etila, diclorometano e água destilada.

\section{Equipamentos}

Serão usados uma balança semi-analítica, uma luminária com lâmpada UV, um evaporador rotativo, um equipamento para determinação de ponto de fusão e um forno de micro-ondas doméstico marca Eletrolux (modelo MEF28 - 18L) adaptado com condensador de refluxo (Figura 1). Tal forno foi baseado no relatado por Ferreira e colaboradores, ${ }^{16}$ consistindo suas adaptações em: (a) tubo em U para circulação de água a fim de remover o excesso da radiação e não danificar o magnetron e; (b) conector de vidro com junta 24/40 na parte superior a fim de ligar o balão ao condensador de refluxo.

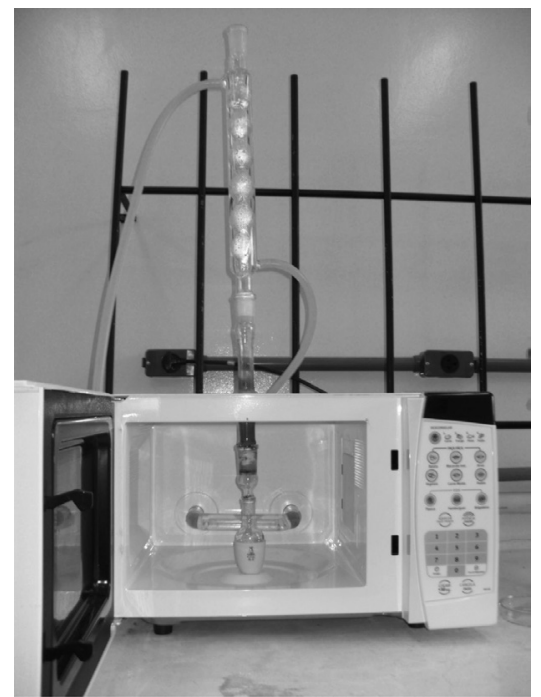

Figura 1. Forno de micro-ondas adaptado usado em nosso laboratório

\section{Caracterização do produto de acoplamento}

No desenvolvimento do referido miniprojeto, o produto do acoplamento catalítico da benzamida com o 4-iodoanisol foi $\mathrm{N}-(4-$ metoxifenil)benzamida, sendo confirmado pelo seu ponto de fusão $\left(152^{\circ} \mathrm{C}\right)$ e por análises de espectroscopia de IV e cromatografia gasosa com espectrometria de massas (CG-EM).

A análise de cromatografia gasosa acoplada à espectrometria de massas foi realizada em um cromatógrafo Shimadzu GC-17A equipado com coluna de DB-1 (dimetilsiloxano) $30 \mathrm{~m}$, diâmetro 0,25 mm. Gás vetor: He (1,5 mL/min) acoplado a um espectrômetro de massas Shimadzu GCMS-QP5050 operando no modo de impacto eletrônico $(70 \mathrm{eV})$. Programação de temperatura: início $100^{\circ} \mathrm{C}$ durante $1 \mathrm{~min}$; aquecimento de $15^{\circ} \mathrm{C} / \mathrm{min}$ até $250^{\circ} \mathrm{C}$ e permanência nessa temperatura por $9 \mathrm{~min}$.

Já a análise de IV foi realizada em espectrômetro PerkinElmer Precisely - Spectrum 100 FT-IR Spectrometer, utilizando a técnica de discos de $\mathrm{KBr}$.

\section{1a aula: $\mathrm{N}$-arilação da benzamida}

Num balão de $25 \mathrm{~mL}$, adicionar $0,121 \mathrm{~g}$ de benzamida ( $1 \mathrm{mmol}$ ); 0,234 g de 4-iodoanisol ( $1 \mathrm{mmol}) ; 0,276 \mathrm{~g}$ de $\mathrm{K}_{2} \mathrm{CO}_{3}(2 \mathrm{mmol})$ como base de Lewis; 0,019 g de CuI (0,1 mmol) como catalisador; 0,018 g de 1,10-fenantrolina $(0,1 \mathrm{mmol})$ como ligante e $8 \mathrm{~mL}$ de DMF (N,N-dimetilformamida) para servir de solvente. Conectar o balão no condensador de refluxo do forno de micro-ondas e iniciar o aquecimento com o tempo determinado pelo professor. Como o reator de micro-ondas utilizado não possui agitação, é necessário interromper o aquecimento para revolver a mistura reacional a cada 20 minutos.

\section{2⿳a aula: Separação do produto}

Lavar o balão da aula anterior com $5 \mathrm{~mL}$ de diclorometano e transferir o líquido para um funil de separação. Repetir a operação usando 5 $\mathrm{mL}$ de água deionizada. Coletar a fase orgânica (inferior) num béquer. Extrair novamente, com diclorometano, a fase aquosa remanescente no funil de decantação a fim de garantir que todo o produto passe para o béquer, assim, adicionar mais $3 \mathrm{~mL}$ do solvente no funil de separação, agitar e separar a fase inferior (juntar no béquer). Para remover traços de água, adicionar, na fase orgânica, uma espátula de $\mathrm{K}_{2} \mathrm{CO}_{3}$ anidro (ou outro sal anidro como $\mathrm{MgSO}_{4}$ ). Transferir o líquido sobrenadante para uma coluna cromatográfica com adsorvente sílica gel 60 (70-230 mesh) a fim de proceder a separação do produto dos reagentes não convertidos. Eluir com $80 \mathrm{~mL}$ de eluente acetato de etila / hexano (3:1), separando em tubos de ensaio frações de $+/-4 \mathrm{~mL}$. Preparar uma pequena placa de cromatografia em camada delgada (cromatoplaca de sílica gel $60 \mathrm{~F}_{254}$ ) de cada tubo de ensaio coletado, eluir com acetato de etila / hexano (1:1) em cuba cromatográfica e revelar em lâmpada UV a fim de determinar o valor dos Rf's encontrados. Determinar, através do mesmo procedimento, os Rf's dos reagentes de partida para, na próxima aula, proceder a separação e identificação do material cromatografado.

\section{3a aula: Purificação e identificação do produto}

Separar os tubos de ensaio que apresentaram os mesmos valores de Rf, sendo diferentes dos valores dos reagentes, e transferir para balão de $250 \mathrm{~mL}$, previamente pesado. Remover o solvente em evaporador rotativo até a secura do balão. Pesar o balão completamente isento de solvente para calcular o rendimento da reação. Determinar o ponto de fusão do sólido obtido.

Nota: Uma alternativa à caracterização do produto obtido é o uso do espectrofotômetro de IV para visualizar a ligação $\mathrm{C}=\mathrm{O}$ e $\mathrm{N}-\mathrm{H}$, bem como da cromatografia gasosa com espectrometria de massas (CG-EM) a fim de encontrar a massa molar do produto obtido, contudo, tais equipamentos não estão disponíveis na maioria dos laboratórios didáticos.

N-(4-metoxifenil)benzamida. Sólido (PF $\left.152{ }^{\circ} \mathrm{C}\right)$. CG-MS (IE, $70 \mathrm{eV}$ ) m/z (\%): 227 (M+, 37), 122 (7), 105 (100), 95 (6), 77 (52), $65(3), 51(13)$. IV $\left(\mathrm{KBr}, \mathrm{cm}^{-1}\right): 3329,15\left(\mathrm{v}_{\mathrm{N}-\mathrm{H}}\right) ; 1645,40\left(\mathrm{v}_{\mathrm{C}=\mathrm{O}}\right)$; $1502,44\left(v_{\mathrm{N}-\mathrm{H}}\right)$.

\section{RESULTADOS E DISCUSSÃO}

\section{Notas explicativas referentes às aulas práticas}

O papel de cada componente no ciclo catalítico para o acoplamento N-C em estudo pode ser observado no Esquema 2.

A opção por aquecimento em micro-ondas foi feita, pois a reação com aquecimento em banho de óleo leva mais de $48 \mathrm{~h}$ para ser completada, assim, com um custo inferior a $\mathrm{R} \$ 400,00$, montou-se um reator para síntese orgânica que trabalha em pressão atmosférica 


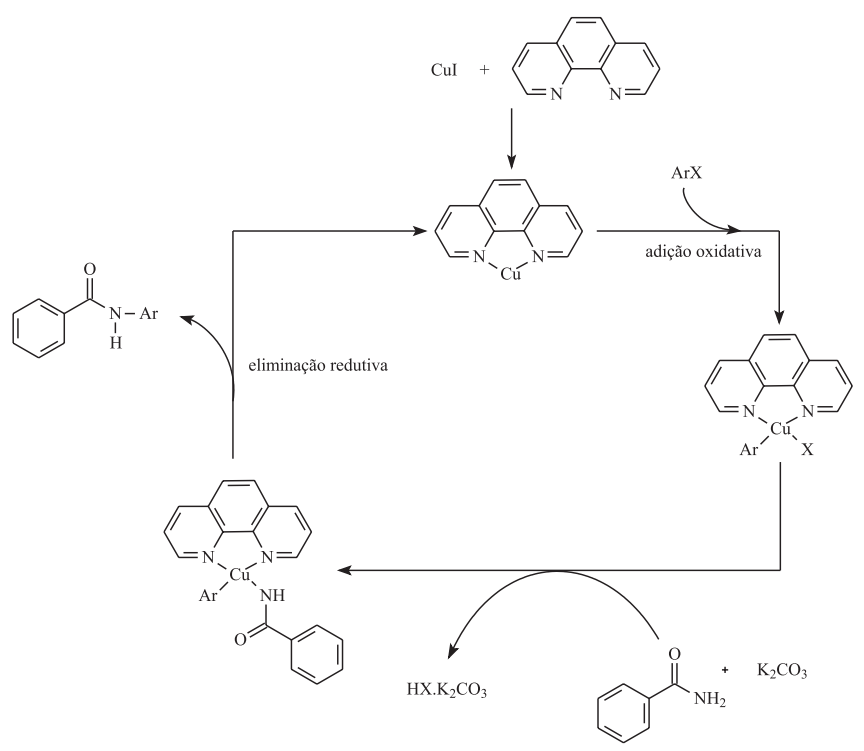

Esquema 2. Possível mecanismo para $\mathrm{N}$-arilação da benzamida via $\mathrm{CuI} /$ 1,10-fenantrolina

conforme o relato de Ferreira e colaboradores. ${ }^{16}$ Tal aparato reduziu drasticamente o tempo reacional, resultando em $97 \%$ de rendimento isolado no produto de acoplamento após 2,5 h de reação.

É interessante o uso de esferas de ebulição no balão para serem centros de nucleação e evitarem a projeção da mistura reacional até o condensador de refluxo. O aquecimento deverá ocorrer, em potência igual ou superior a $80 \%$, por no mínimo $1 \mathrm{~h} 30 \mathrm{~min}$. É interessante que o professor peça para os grupos fazerem a reação com tempos diferenciados a fim de traçar um paralelo do tempo reacional com o rendimento obtido.

Após o término do tempo reacional, o balão contendo a mistura reacional (líquido com precipitado) deverá ser guardado fechado, em temperatura ambiente e ao abrigo da luz, para posterior análise no próximo encontro de laboratório.

Durante a $2^{\mathrm{a}}$ aula (separação do produto), aplicada no Curso Técnico em Química do Colégio Dom Feliciano, foi substituída a coluna cromatográfica na separação por uma placa de cromatografia em camada delgada com dimensões $10 \times 20 \mathrm{~cm}$, aplicando várias vezes o extrato bruto concentrado (em linha). A mancha com Rf correspondente ao produto foi raspada e dissolvida em diclorometano, sendo filtrada e evaporada. Tal procedimento foi escolhido pela simplicidade e menor tempo empregado.

$\mathrm{O}$ valor do Rf da benzamida determinada pelos alunos ( $3^{\mathrm{a}}$ aula) deve ser próximo a 0,20 , já o 4-iodoanisol tem $\mathrm{Rf}=0,77$. No teste da referida aula prática, nosso grupo encontrou para o produto de acoplamento um Rf que oscilava de 0,50 a 0,70 correspondente a uma mancha arrastada. Espera-se, se a reação ocorrer completamente, obter $1 \mathrm{mmol}$ de produto, ou seja, 0,227 g. Para garantir que o sólido obtido realmente se trata do produto de acoplamento catalítico, o ponto de fusão deverá ser avaliado. Espera-se encontrar ponto de fusão próximo a $152^{\circ} \mathrm{C}$, valor esse que difere da benzamida (PF de 124-127 ${ }^{\circ} \mathrm{C}$ ) e do 4-iodoanisol ( $\mathrm{PF}$ de $47-50^{\circ} \mathrm{C}$ ), tornando essa técnica de caracterização simples bastante confiável.

\section{Dados coletados durante o miniprojeto}

Com os resultados referentes à técnica cromatográfica empregada, rendimento da reação e ponto de fusão do produto de acoplamento, passados ao professor através do relatório de aula prática, foi possível construir a Tabela 1 e um gráfico correlacionando o tempo reacional com o rendimento obtido (Figura 2).
Tabela 1. Dados coletados após entrega de relatório do miniprojeto

\begin{tabular}{ccccc}
\hline Reação & Tempo (min) & $\begin{array}{c}\text { Técnica cro- } \\
\text { matográfica }\end{array}$ & $\begin{array}{c}\text { Rendimento } \\
(\%)\end{array}$ & $\begin{array}{c}\text { Ponto de } \\
\text { fusão }\left({ }^{\circ} \mathbf{C}\right)\end{array}$ \\
\hline 1 & 60 & $\begin{array}{c}\text { Camada } \\
\text { delgada }\end{array}$ & 46 & 150 \\
2 & 60 & $\begin{array}{c}\text { Camada } \\
\text { delgada }\end{array}$ & 41 & 154 \\
3 & 80 & $\begin{array}{c}\text { Camada } \\
\text { delgada }\end{array}$ & 54 & 151 \\
4 & 80 & $\begin{array}{c}\text { Camada } \\
\text { delgada }\end{array}$ & 50 & 151 \\
5 & 90 & $\begin{array}{c}\text { Camada } \\
\text { delgada }\end{array}$ & 53 & 152 \\
6 & & & 57 & 150 \\
7 & 100 & $\begin{array}{c}\text { Coluna } \\
\text { Coluna }\end{array}$ & 62 & 150 \\
8 & 120 & Coluna & 70 & 153 \\
9 & 120 & Coluna & 48 & 151 \\
10 & 120 & Coluna & 17 & 151 \\
11 & 150 & Coluna & 97 & 152 \\
\hline
\end{tabular}

As reações 1 a 5 foram realizadas pelos alunos do Curso Técnico em Química do Colégio Dom Feliciano, sendo realizadas em tempos de 60 a 90 minutos. A escolha do tempo baixo e da técnica de cromatografia em camada delgada para separação do produto formado foi baseada em dois fatores: (a) limitação de tempo para fazer o experimento; e (b) simplicidade da técnica cromatográfica. Contudo, os resultados foram satisfatórios em termos de rendimento e pureza obtida.

Já as reações de 6 a 10 foram realizadas pelos alunos do Curso de Engenharia Agroindustrial da FURG e, como a disponibilidade de tempo para o experimento era maior, trabalhou-se com tempos reacionais de 90 a 120 minutos. Também se optou por realizar a separação por técnica de cromatografia em coluna já que a mesma está na ementa da disciplina Química Orgânica Experimental. As reações 6,7 e 8 mostraram um resultado esperado para um tempo reacional maior aliada a uma técnica de separação mais confiável. Contudo, os grupos de laboratório que conduziram as reações 9 e 10 procederam a separação incompleta, visto que os rendimentos obtidos divergem do resultado da reação 8, que apresentou o mesmo tempo reacional.

E, finalizando a análise da Tabela 1 , verifica-se que a reação estudada se completa num tempo de 150 minutos sendo isolado o produto com excelente rendimento pela técnica de cromatografia em coluna (reação 11). Tal reação serviu de base para a referida investigação sendo realizada pelos alunos bolsistas de iniciação científica que trabalharam no projeto. A avaliação da necessidade de catalisador (CuI) e ligante (1,10-fenantrolina) também fora realizada nas mesmas condições da reação 11, sendo monitorada por cromatografia gasosa. Assim, na ausência de CuI não houve formação do produto de acoplamento e, num ensaio com $\mathrm{CuI}$ na ausência de ligante, apenas observou-se um rendimento de $6 \%$ em N-(4-metoxifenil)benzamida, corroborando a importância de ambos.

Analisando o gráfico (Figura 2) observa-se a dependência do rendimento frente ao tempo reacional. Obviamente, retirando-se os resultados contraditórios no tempo de 120 minutos (reações 9 e 10), verifica-se uma tendência de aumentar o rendimento com a elevação do tempo reacional. A opção em trabalhar com diferentes tempos reacionais foi adotada para, no fechamento do miniprojeto, os alunos confirmarem visualmente a referida tendência ilustrada na Figura 2.

Nas ementas de disciplinas de Química Orgânica Experimental o tópico síntese orgânica via acoplamento catalítico é raro, principalmente, para alunos que não cursam graduação em química. Para alunos de cursos técnicos em química isso nem sequer é mencionado. Com o objetivo de suprir essas lacunas criamos o referido miniprojeto, sendo 


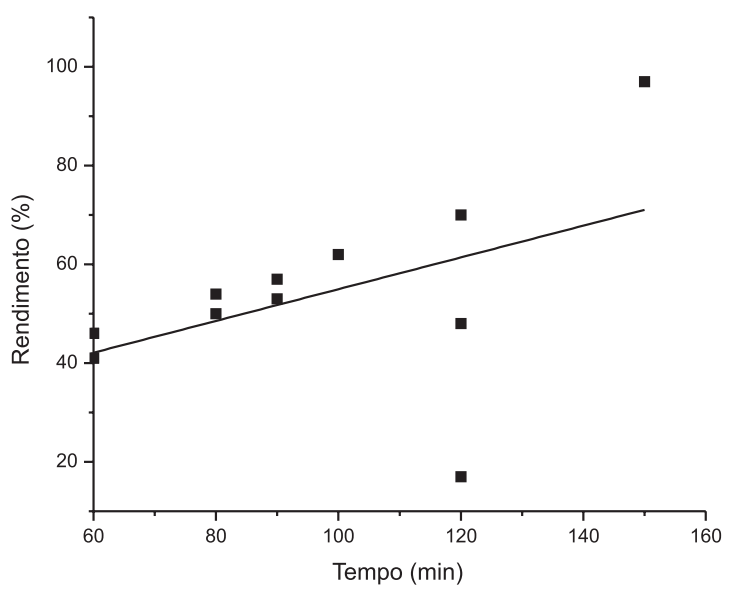

Figura 2. Correlação entre tempo reacional e rendimento isolado na reação estudada

que o mesmo, além de tratar do tema acoplamento catalítico, aborda as importantes técnicas de cromatografia em camada delgada e em coluna, faz uso do evaporador rotativo e do forno de micro-ondas adaptado. Embora os alunos não tenham participado da adaptação do forno de micro-ondas, o seu emprego foi agente motivador, tornando-se peça fundamental no desenvolvimento do miniprojeto. Como o fator custo é de suma importância no desenvolvimento de uma aula prática, sem o uso do micro-ondas ter-se-ia a necessidade de usar catalisadores mais eficientes (derivados de paládio) para ter um tempo reacional compatível com a aula de laboratório, o que deixaria o processo oneroso.

Simoni ${ }^{17}$ propõem uma classificação para os experimentos de laboratório em Química em quatro categorias e definiu cada uma como descritas, de forma resumida, a seguir: conceituais (C) - envolvem conceitos fundamentais da química; metodológicos (M) - têm como objetivo central o desenvolvimento do aluno em técnicas experimentais; fundamentais $(F)$ - envolvem medidas de quantidades fundamentais para a caracterização de um sistema químico e, quantitativos (Q) - envolvem determinações quantitativas de diversos gêneros.

Ainda segundo o autor, ${ }^{17}$ alguns experimentos podem ser classificados em mais de uma categoria, inclusive existindo experimentos que podem receber todas as classificações propostas. ${ }^{18}$ Foi sugerido também pelos autores que uma distribuição dos experimentos dentro das quatro categorias é desejável.

Tal classificação foi aplicada no miniprojeto aqui apresentado, sendo que as diferentes etapas podem ser classificadas como mostrado a seguir.

Os tópicos que abordam uso de vidrarias para medidas volumétricas (1 ${ }^{\underline{a}}, 2^{\underline{a}}$ e $3^{\underline{a}}$ aulas), uso da balança (1 $1^{\underline{a}}$ e $3^{\underline{a}}$ aulas), uso do termômetro ( $3^{\underline{a}}$ aula), uso do micro-ondas adaptado (1 $1^{\mathrm{a}}$ aula) e separação de misturas ( $2^{\underline{a}}$ aula) permitem classificar o experimento como metodológico (M).

O conceito de reação química é abordado na $1^{\mathrm{a}}$ aula, sendo observada visualmente pela troca de coloração e confirmada após análise cromatográfica na $2^{\underline{a}}$ aula. Por isso, o experimento pode ser classificado como conceitual (C).

O cálculo de rendimento da reação do produto isolado realizado na $3^{3}$ aula é uma atividade típica de experimento quantitativo (Q).

No miniprojeto não são executadas atividades para determinação de constantes fundamentais, o que não permite a classificação como experimento fundamental $(\mathrm{F}) .{ }^{17}$

\section{CONCLUSÃO}

A N-arilação da benzamida com 4-iodoanisol se mostrou um tema apropriado para o ensino de Química Orgânica Experimental, tanto em nível de graduação quanto em nível técnico. A realização das atividades incluídas no miniprojeto permitiu o desenvolvimento de habilidades e conceitos presentes nos componentes curriculares destas disciplinas, podendo o mesmo ser classificado como experimento conceitual (C), metodológico (M) e quantitativo (Q). O sistema catalítico mostrado já é bem estudado no Laboratório de Síntese Orgânica Catalítica - LSO[Cat] - da FURG, mostrando ser eficiente para outras moléculas nitrogenadas como imidazol, indol e lofina dando bons rendimentos. A escolha do 4-iodoanisol para esse miniprojeto reside no fato de ser o iodeto de arila $p$-substituído que apresenta maior dificuldade de ser acoplado, dessa forma, outros ArI, se empregados, fornecerão rendimentos melhores.

\section{AGRADECIMENTOS}

Ao CNPq pelo apoio financeiro (edital CNPq/VALE S.A. $\mathrm{N}^{\circ}$ 05/2012). M. Dalmás, C. L. Ferreira, J. A. O. dos Santos e T. K. Bolzan também agradecem ao $\mathrm{CNPq}$ pelas bolsas recebidas.

\section{REFERÊNCIAS}

1. McClenaghan, N. D.; Passalacqua, R.; Loiseau, F.; Campagna, S.; Verheyde, B.; Hameurlaine, A.; Dehaen, W.; J. Am. Chem. Soc. 2003, 125,5356

2. Hassan, J.; Sevignon, M.; Gozzi, C.; Schulz, E.; Lemaire, M.; Chem. Rev. 2002, 102, 1359.

3. Kunz, K.; Scholz, U.; Ganzer, D.; Synlett 2003, 15, 2428.

4. Antilla, J. C.; Baskin, J. M.; Barder, T. E.; Buchwald, S. L.; J. Org. Chem. 2004, 69, 5578.

5. Saeedi, A; Zaman, S.; Jamil, M.; Mirza, B.; Turk. J. Chem. 2008, 32, 585.

6. Young, D. H.; Lewandowski, V. T.; Plant Physiol. 2000, 124, 115.

7. Pani, L.; Gessa, G. L.; Mol. Psychiatry 2002, 7, 247.

8. Assad, L.; Ciência \& Cultura 2012, 64, 6.

9. de Souza, R. O. M. A.; Miranda, L. S. M.; Quim. Nova 2011, 34, 497.

10. Cunha, S.; Lustosa, D. M.; Conceição, N. D.; Fascio, M.; Magalhães, V.; Quim. Nova 2012, 35, 638.

11. Cunha, S.; de Santana, L. L. B.; Quim. Nova 2012, 35, 642.

12. Teixeira, E. F.; dos Santos, A. P. B.; Bastos, R. S.; Pinto, A. C.; Kümmerle, A. E.; Coelho, R. R.; Quim. Nova 2010, 33, 1603.

13. Konrath, E. L.; Piedade, M.; Eifler-Lima, V. L.; Quim. Nova 2012, 35, 1887.

14. O referido trabalho foi aplicado na disciplina de Química Orgânica Experimental dos cursos de Engenharia Agroindustrial: Agroquímica e Engenharia Agroindustrial: Indústrias Alimentícias, da Universidade Federal do Rio Grande (FURG), bem como, na disciplina de Química Orgânica III do Curso Técnico em Química do Colégio Dom Feliciano (Gravataí). A parceria da FURG com o Colégio Dom Feliciano já é antiga e, nesse projeto, fora oficializada pela aprovação da chamada CNPq/VALE S.A. No 05/2012 - Forma-Engenharia, que tem por objetivo estimular a formação de engenheiros no Brasil motivando alunos de Ensino Médio e Técnico, através da cooperação entre escola e universidade, a seguir nessa área do conhecimento.

15. Peixoto, C. R. M.; Rosa, G. R.; Silva, C. N.; Santos, B. T.; Engelmann, T. L.; Quim. Nova 2012, 35, 1686.

16. da Silva, F. C.; Ferreira, V. F.; de Souza, M. C. B. V.; Quim. Nova 2006, $29,376$.

17. Simoni, D. A.; Andrade, J. C.; Simoni, J. A.; Quim. Nova 2011, 34, 1818.

18. Simoni, D. A.; Andrade, J. C.; Faigle, J. F. G.; Simoni, J. A.; Quim. Nova 2002, 25, 1034. 